Special Issue: Biological effects of space radiation -Part I-

\section{LOH Analyses for Biological Effects of Space Radiation: Human Cell Culture in "Kibo" of International Space Station}

\author{
Fumio Yatagai ${ }^{1,5, \dagger}$, Akihisa Takahashi ${ }^{2}$, \\ Masamitsu Honma ${ }^{3}$, Hiromi Suzuki ${ }^{4,6}$, \\ Katsunori Omori ${ }^{5}$, Masaya Seki ${ }^{6,7}{ }_{4}$ \\ Toko Hashizume ${ }^{6,7}$, Toru Shimazu ${ }^{4}$, \\ Shuichi Enomoto', Takeo Ohnishi ${ }^{2}$ \\ and Noriaki Ishioka ${ }^{5,7}$ \\ ${ }^{1}$ RIKEN Institute, 2-1 Hirosawa, Wako-shi, Saitama 351- \\ 0198, Japan \\ ${ }^{2}$ Nara Med. Univ. 840 Shijyo-cho, Kashihara, Nara 634- \\ 8521, Japan \\ ${ }^{3}$ Natl. Inst. Health Sci., 1-18-1 Kami-yoga, Setagaya-ku, \\ Tokyo 158-8051, Japan \\ ${ }^{4}$ Japan Space Forum, 2-2-1 Otemachi, Chiyoda-ku, \\ Tokyo 104-0004, Japan \\ ${ }^{5}$ Japan Aerospace Exploration Agency, 2-1-1 Sengen, \\ Tsukuba-shi, Ibaraki 305-8505, Japan \\ ${ }^{6}$ Kagoshima Univ. Grad. Sch. 8-35-1 Sakuragaoka, \\ Kagoshima-shi, Kagoshima 890-8544, Japan \\ ${ }^{7}$ Adv. Engineering Services, 1-9-13 Yaesu, Chuo-ku, \\ Tokyo 103-0028, Japan
}

\section{Abstract}

This is a review paper to introduce how our developing methodology for loss of heterozygosity (LOH) analysis is predictable to detect the biological effects of space radiation. An experiment was successfully performed at 'Kibo' facility of International Space Station (ISS) from November 2008 to March 2009. If the field is restricted to life science, this is the first experiment at 'Kibo'. In this first experiment, we designed to elucidate the following influences of space-radiation and microgravity in human lymphoblastoid cell, TK6: i) a mutagenic effect of space radiation, ii) an effect of microgravity on mutation induction, and $\mathrm{iii)}$ a radioadaptive response after recovering the sample to the earth. Since the cells were successfully brought back to the earth at the beginning of April, we just started to analyze mutagenic effects of the frozen cells which were exposed to space radiation during about 4 months and half preservation at 'Kibo', determine influences of microgravity effects on induction of mutations

Received: April 17, 2009; Accepted: May 13, 2009

Review Article

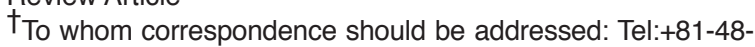
467-9710; Fax:+81-48-467-9710; E-mail: yatagai@riken.jp by analyzing the cells incubated during 8 days under $1 G$ or $\mu G$ at 'Kibo', and evaluate a potential ability of the above frozen-cells to induce a radioadaptive response by measuring the repair efficiencies of DNA double-strand breaks introduced after recovering the sample to the earth. (C2009 Jpn. Soc. Biol. Sci. Space; Article ID: 092301002

\section{Introduction}

It is important to estimate human health risks for persons occupationally exposed to ionizing radiation (IR), such as air line crews and radiation workers in medical and industrial fields. Such risk for the astronauts exposed to space radiation is a typical example, because the radiation background in space is considered to be more than 100-fold higher than the level of the earth. Biological effects of space radiation can be classified into the following two-types, direct effects and indirect effects (Fig. 1). Direct effects are considered to be mainly due to DNA damage caused by IR. On the other hand, indirect effects are well reflected in the phenomena, known as adaptive response, bystander effect, genome instability etc. Using a loss of heterozygosity ( $\mathrm{LOH}$ ) analysis technique, we plan to detect direct effects as chromosomal changes caused by DNA damage. For detection of indirect effects, we focus on radioadaptive response, which is defined as an acquired resistance of cells exposed to challenging IR by an additional priming low-dose IR exposure. Here, our approach to the adaptive-response study is based upon the assumption that the cells were exposed to space radiation during the long preservation with a dose corresponding to that of a priming exposure.

Fortunately we have obtained an opportunity to investigate influences of space radiation in human lymphoblastoid cells TK6, which are brought back to the earth after about 130 days preservation in ISS. We started to investigate effects of space radiation, based upon the assumption that the space-radiation caused DNA damage can be accumulated in the frozen cells and detected as a mutation by the post-flight analyses. Furthermore, we are now making an attempt to detect microgravity effects on mutation induction in the cells, which were incubated in space. The details of experimental approach are described below.

\section{An outline of ISS experiment}

The outline of ISS experiment for elucidating biological influences of space radiation and microgravity is schematically illustrated in Fig. 2. To detect not only the radiation effects but also the microgravity effect, we brought the frozen cells of human lymphoblastoid TK6 to ISS and to incubate a part of cell preparation during 8 days under $1 \mathrm{G}$ or $\mu \mathrm{G}$ condition. The $1 \mathrm{G}$ condition was created by using the relatively small rotor $(1.0-1.5 \mathrm{G})$ in the Cell Biology Experiment Facility (CBEF) on ISS (Fig. 3) (Ishioka et al., 2004). The cells were then re-frozen on 


\section{Space Radiation Exposure}

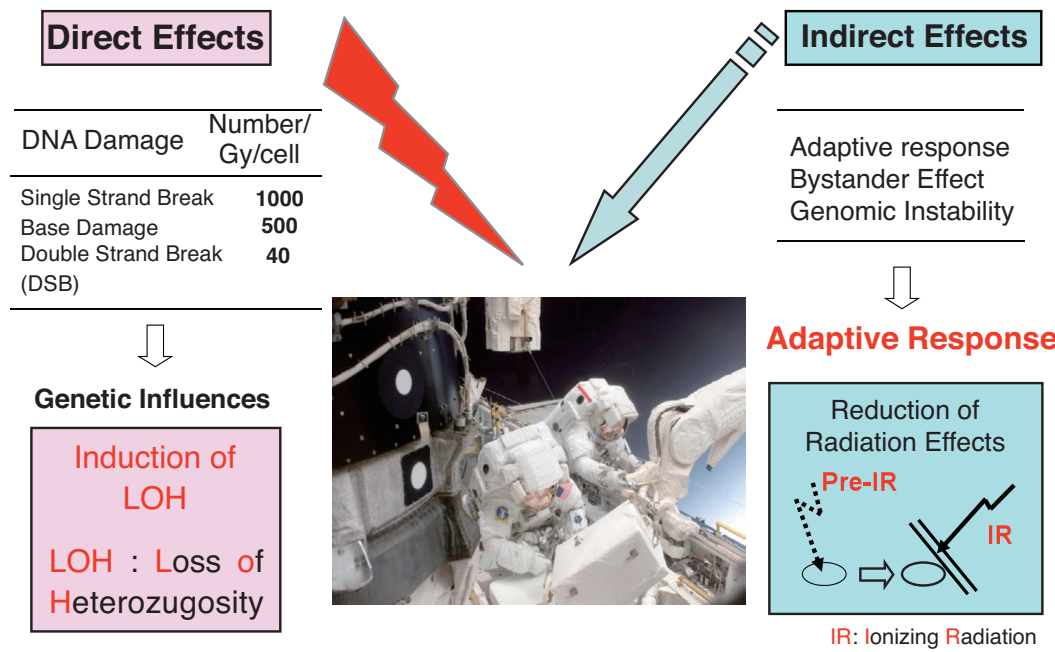

Fig. 1. Biological influences of space radiation

Biological effects of space radiation exposure can be classified into these two types, direct effects and indirect effects (see text).

ISS by the astronauts, and recently brought back to the earth. Both assays for mutation and adaptive-response were now under the study on the ground (Fig. 2). Since this experiment requires a variety of well-designed cell handling techniques, e.g. freezing before the launch, thawing and refreezing on ISS, growing on ISS, reincubating after the recovery, we have made lots of efforts to achieve a reproducible result of cell preparation by ground-simulation experiments using the CBEF ground model and simulated cell freezing system for ISS freezer (MELFI). Before the launch, the crew training was also performed at Tsukuba Space Center.

\section{Methodologies for analyses of space-radiation effects}

\section{LOH analysis for mutagenic effects}

The experimental procedures for exposure of TK6 cells to ionizing radiation and selection of thymidine kinase deficient ( $\left.\mathrm{TK}^{-}\right)$mutants are shown in Fig. 4. The details concerning these procedures were already described in our previous paper (Morimoto et al., 2002a, Morimoto et al., 2002b, Umebayashi et al., 2005, Umebayashi et al., 2007). Briefly, the selected $\mathrm{TK}^{-}$mutants were analyzed to determine the loss of $T K$ heterozygosity (LOH) using PCR amplification of the exon 4 and 7 regions of the TK locus (Honma et al., 1997 \& 2000). The outline of this step of analysis is schematically illustrated in Fig. 5. Further characterization was also applied for the LOH mutants to determine the extent of deleted or replaced region of chromosome by PCR amplification of the 11 satellite markers on chromosome 17 (Yatagai et al., 2004). Although the results of this chromosome mapping were not shown in this review, the observed interstitial-deletion seemed to be specific to radiation and they were considered to be useful for detection of space radiation effects.
$D S B$-repair evaluation for detection of adaptive response

As reported by many investigators, the radioadaptation can be identified as the decreased frequency of chromosomal aberrations (Ikushima 1987, Rigaud et al. 1993, Zhou et al., 1993, Azzam et al., 1994, etc). Since genetic changes at chromosome level are considered to be caused by chromosome break or DNA double-strand break (DSB), the repair of DSB was evaluated by our model system (Fig. 6) (Honma et al., 2003). The repair of DSBs is considered to be mainly due to two pathways, non homologous end-joining (NHEJ) and homologous recombination (HR). The details of methodologies for evaluating the above DSB repair were already described in our previous paper (Honma et al., 2003, Honma etal., 2007, and Yatagai et al.,2008b). Briefly, an 18 bp sequence containing the recognition sequence for the restriction enzyme I-Scel was introduced at the region upstream of exon 5 in the $T K^{+}$allele of TK6 cells. The cells containing this insert were designated TSCE5. In addition, a compound heterozygote (TK-/-) cell line, TSCER2 was obtained by a spontaneous reversion in a TSCE5 cell clone ( $G$ to $A$ in the 23 rd base position of exon 5). When a DSB at the I-Scel site is repaired by NHEJ in TSCE5, it causes a deletion, permitting the cell to be isolated as a TK-deficient mutant. In TSCER2, on the other hand, when the DSB is repaired by HR between $\mathrm{TK}$ alleles, a $\mathrm{TK}^{+}$allele is generated resulting in a revertant phenotype.

\section{Expected results}

Mutagenic effects of space radiation

A higher background of ionizing radiation including a variety of heavy charged particles is present in a space environment. However, we need to utilize the radiation-sensitive methodology to detect the genetic 


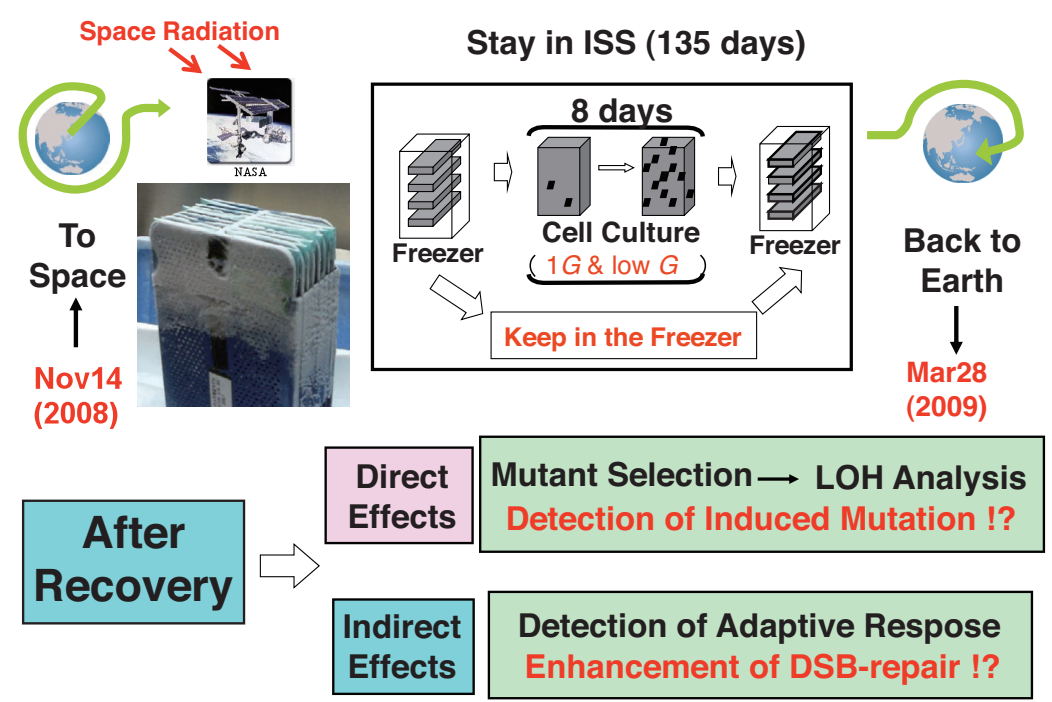

Fig. 2. A plan of space experiment using "Kibo" facility The plan of ISS experiments for studying the biological influences of space radiation and microgravity is schematically illustrated in this figure (see text).

influences of such high background radiation, because the accumulated dose of space radiation after 4 months and half stay on ISS can be estimated to be 35 70 mGy. Taking the expected dose into consideration, we have designed a ground simulation experiment using the frozen cells for sensitive detection of mutation (Fig. $5 a)$. According to the results of simulation experiment, the sensitivity of mutation detection for the frozen cells was lower than the case of suspension culture, but the detection was suggested to be possible even after the one month preservation at $-80^{\circ} \mathrm{C}$ (Morimoto et al., 2002b and Umebayashi et al., 2005). Similarly to the case of suspension culture, we frequently observed the specific pattern of hemizygous $\mathrm{LOH}$ (interstitial deletion), which seemed to be characteristic to radiation exposure (data, not shown). Furthermore, the low-dose radiation effects were clearly observed in the following ground simulation

\section{Cell Biology Experiment Facility: CBEF}

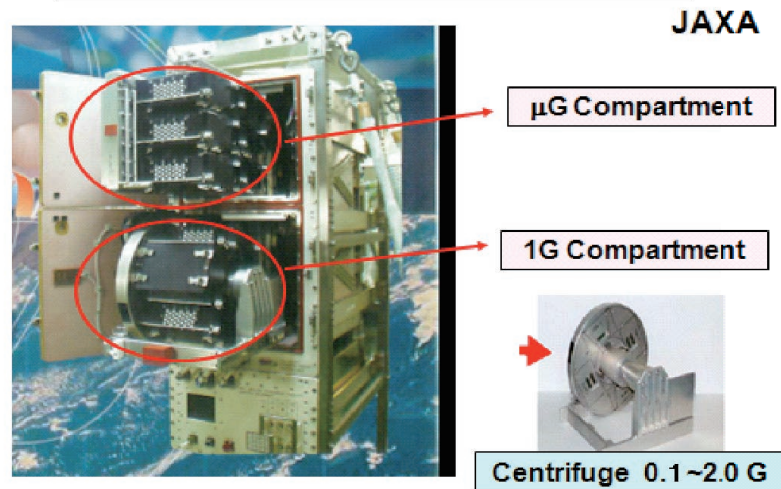

Fig. 3. Cell culture system in Kibo (CBEF)

The $1 \mathrm{G}$ condition is created by using the small rotor $(1.0-1.5 \mathrm{G})$ in the Cell Biology Experiment Facility (CBEF) in Kibo of ISS (Ishioka et al., 2004). experiments. One is designed for detecting the mutation induction after low-dose/low-dose-rate $\mathrm{Y}$-rays (Fig. 5b)) (Umebayashi et al., 2007), and another is examined for the possibility of adaptation that the low-dose priming $X$-ray irradiation reduces the mutation induction after challenging X-ray irradiation (Fig. 5c)) (Yatagai et al., 2008a). As a summary, these simulations support the expectation that we could detect mutagenic effects of space radiation in this experimental design.

Here, we would like to emphasize the following point. Based upon the assumption that radiation damage in the frozen cells can be accumulated during the whole ISS stay, we could detect the mutagenic effects of space radiation as mentioned above.

\section{Microgravity effect}

For estimation of human health risk in space, we would like to evaluate influences of microgravity on the mutation induction in the human cultured cells growing under the high radiation-background in space. Here, we designed to compare the mutation frequencies, which were determined by the above TK mutational analyses for both cells incubated under $\mu G$ and $1 G$ for 8days. This comparison could reflect an effect of microgravity on mutation induction, because both cells had been exposed to space radiation with the same dose before the period of incubation on ISS. This is the reason why we planned to incubate the cells just before the recovery of cells to the earth. Compared to our proposed plan, the return flight of space shuttle was delayed in about one month, but the incubation period was still close to the end of long stay. The comparison also might predict a possibility of the combined effects of radiation and microgravity, because the effect of only radiation can be estimated from the comparison between the flight and ground control frozen cells. As far as the combined effects, there are a variety of reports using different biological systems, synergistic, or additive or no additive, etc. We hope that 

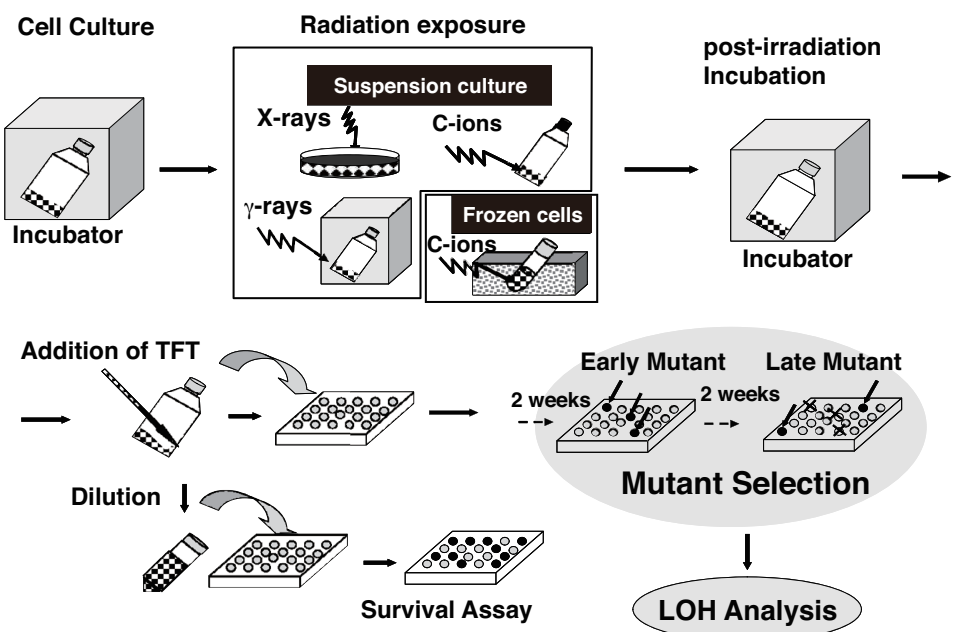

Fig. 4. A general procedure for isolation of TK mutant

Following the ionizing radiation exposure, the TK mutant clones were selected as resistant to $4 \mu \mathrm{g} / \mathrm{ml}$ trifluorothymidine (TFT). Early TK mutant clones (EMs) and late TK mutant clones (LMs) were selected separately after two and four weeks of incubation in this selection medium, respectively. The selected TK mutants were classified into non LOH, hemizygous LOH or homozygous LOH by LOH analysis (see text).

our space experiment becomes an important occasion from the view point of microgravity effects.

\section{An adaptive response}

The $\mathrm{LOH}$ analysis was also applied for the X-ray to X-ray 'radioadaptive response' experiments (Fig. 5c). TK6 cells were pre-irradiated with low-dose (5 cGy) of X-ray, and then exposed to challenging dose (2 Gy) of X-rays after $6 \mathrm{hr}$ incubation. The TK mutation frequency after $2 \mathrm{~Gy} X$-ray exposure was reduced in response to the preexposure dose from $18.3 \times 10^{-6}$ to $11.4 \times 10^{-6}$, representing a reduction to approximately $62 \%$ of the non-pretreated or non-conditioned cells (Yatagai et al., 2008a). Under this optimum condition of adaptive response, we have analyzed the influence of priming X-ray exposure on DSB repair by the methodology as mentioned above. The NHEJ repair of DSB was barely influenced by preirradiation of the cells. DSB repair by HR, in contrast, was enhanced by $\sim 70 \%$ after pre-irradiation of the cells under these conditions (unpublished data). Using the frozen cells, we carried out the simulation experiment where the priming low-dose $\mathrm{C}$-ion irradiation was considered to correspond to the space radiation (Fig. 6). In this simulation, we determined the influence of this priming irradiation on repair of DSB. When the cells were preirradiated with 5 cGy of $\mathrm{C}$-ion, they demonstrated a similar tendency of enhancement in HR repair of I-Scel break as shown in the table of Fig. 6.

If the cells recovered from the space are irradiated with the challenging dose of X-rays, for example, 2Gy, we might observe a mutagenic adaptive-response, reduction in mutation induction compared to the ground control. Similarly, the enhanced DSB-repair in the recovered cells compared to that of ground control also seems to be predictable in our system. For detecting a potential ability of adaptive response in the cells preserved as frozen for whole ISS stay, we decided to make both types of assays, mutation induction and DSB repair. Our expectation for a possible detection of adaptive response is also based upon the assumption that radiation damage in the frozen cells can be accumulated during the whole ISS stay.

\section{Acknowledgments}

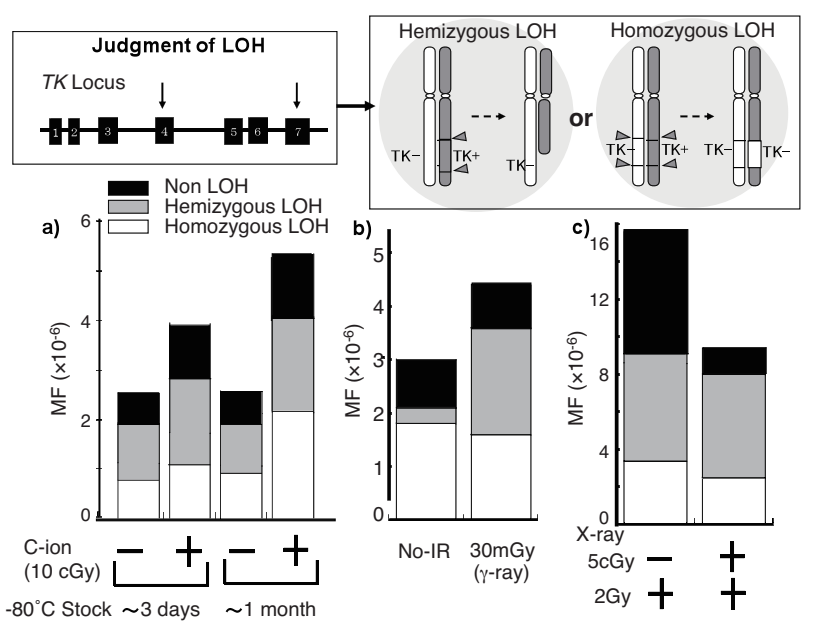

Fig. 5. Judgment of LOH for TK mutant (Distribution of mutational classes)

The selected TK mutants were analyzed to determine the loss of TK heterozygosity (LOH) using PCR amplification of the exon 4 and 7 regions of the TK locus (see text, Honma et al., 1997 \& 2000). The selected TK mutants were classified into non LOH, hemizygous LOH or homozygous LOH by this judgment. The outline of this step of analysis is schematically illustrated in the top panel of this figure. The summary results for these analyses are separately shown in each case; (a) the frozen cells are exposed to $10 \mathrm{cGy}$ carbon ion beam, (b) the cells are cultured in the incubator during the low-dose/low-dose-rate $\gamma$-rays $(30 \mathrm{mGy}$ : $1.2 \mathrm{mGy} / \mathrm{h}$ ), and (c) the cells are exposed to X-ray for detection of an adaptive response ( 5 cGy for priming exposure and 2 Gy for challenging exposure). 


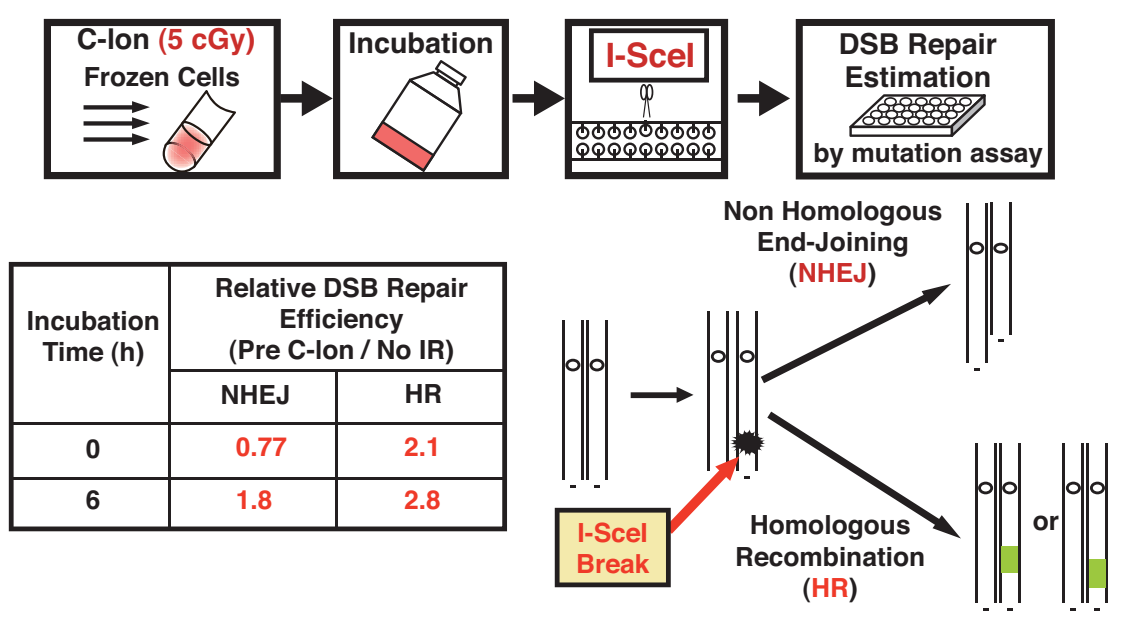

Fig. 6. A space simulation experiment for detecting adaptive response

The strategy of space simulation experiments are shown in the top panel of this figure; (A) The C-ion exposure in the first step is assumed to correspond to the IR exposure in space and the later steps are designed to determine the repair efficiencies of DNA double strand breaks (DSBs), introduced by the I-Scel expression vector. The repair of DSBs is considered to be mainly due to two pathways shown in the bottom panel, non homologous end-joining (NHEJ) and homologous recombination (HR). As a principle for determination of DSB repair efficiency, we measure the TK mutants generated through NHEJ or TK ${ }^{+}$revertants generated through HR, respectively. The representative data obtained with this system is shown in the inserted table.

The authors greatly appreciate Professor Takeo Ohnishi and Dr. Akihisa Takahashi of Nara Prefecture Medical University for providing us a chance to describe this review paper.

\section{References}

Azzam, E.I., Raaphorst, G.P., and Mitchel, R.E. (1994) Radiation-induced adaptive response for protection against micronucleus formation and neoplastic transformation in $\mathrm{C} 3 \mathrm{H} 10 \mathrm{~T} 1 / 2$ mouse embryo cells, Radiat. Res., 138, S28-31.

Honma, M., Zhang, L. S., Hayashi, M., Takeshita, K., Nakagawa, Y., Tanaka, N. and Sofuni T. (1997) Illegitimate recombination leading to allelic loss and unbalanced translocation in p53-mutated human lymphoblastoid cells, Mol. Cell. Biol., 17, 4774-4781.

Honma, M., Momose, M., Tanabe, H., Sakamoto, H., Yu, Y., Little, J.B., Sofuni, T. and Hayashi, M. (2000) Requirement of wild-type p53 protein for maintenance of chromosomal integritym, Mol. Carcinog., 28, 203214.

Honma, M., Izumi, M., Sakuraba, M.,Tadokoro, S., Sakamoto, H., Wang, W., Yatagai, F. and Hayashi, M. (2003) Deletion, rearrangement, and gene conversion; genetic consequences of chromosomal double-strand breaks in human cells, Environ. Mol. Mutagen., 42, 288-298.

Honma, M., Sakuraba, M., Koizumi, T., Takashima, T., Sakamoto, H. and Hayashi, M. (2007) Nonhomologous end-joining for repairing l-Scel induced DNA double strand breaks in human cells, DNA Repair, 6, 781-788.
Ishioka, I., Suzuki, H., Asashima, M., Kamisaka, S., Mogami, Y., Ochiai, T., Aizawa-Yano, T., Higashibata, A., Ando, N., Nagase, M., Ogawa. S., Shimazu, T., Fukui, K. and Fujimoto, N. (2004) Development and verification of hardware for life science experiments in the Japanese experiment module "Kibo" on the International Space Station, J. Gravitat. Physiol., 11, 81-91.

Ikushima, T. (1987) Chromosomal response to ionizing radiation reminiscent of an adaptive response in cultured Chinese hamster cells, Mutat. Res., 180, 215221.

Morimoto, S., Kato, T., Honma, M., Hayashi, M., Hanaoka, F. and Yatagai, F. (2002a) Detection of genetic alterations induced by low-dose $X$ Rays: Analysis of loss of heterozygosity for TK mutation in human lymphoblastoid cells, Radiat. Res., 157, 533538.

Morimoto, S., Honma, M. and Yatagai, F. (2002b) Sensitive detection of $\mathrm{LOH}$ events in a human cell line after C-ion beam exposure, J. Radiat. Res., 43 (Suppl.), S163-167.

Umebayashi, Y., Honma, M., Abe, T., Ryuto, H., Suzuki, H., Shimazu, T., Ishioka, N., Iwaki, M. and Yatagai, F. (2005) Mutation induction after low-dose carbon-ion beam irradiation of frozen human cultured cells, Biol. Sci. Space, 19, 237-241.

Umebayashi, Y., Honma, M., Suzuki, M., Suzuki, H., Shimazu, T., Ishioka, N., Iwaki, M. and Yatagai, F. (2007) Mutation induction in cultured human cells after low-dose and low-dose-rate $\gamma$-ray irradiation: Detection by LOH analysis, J. Radiat. Res., 48, 7-11.

Rigaud, O., Papadopoulo, D. and Moustacchi, E. (1993) Decreased deletion mutation in radioadapted human lymphoblast, Radiat. Res., 133, 94-101. 
Yatagai, F., Morimoto, S., Kato, T. and Honma, M. (2004) Further characterization of loss of heterozygosity enhanced by $p 53$ abrogation in human lymphoblastoid TK6 cells: disappearance of endpoint hotspots, Mutat. Res., 560, 133-145.

Yatagai, F., Umebayashi, Y., Honma, M., Sugasawa, K., Takayama, Y. and Hanaoka, F. (2008a) Mutagenic radioadaptation in a human lymphoblastoid cell line, Mutat. Res., 638, 48-55.

Yatagai, F., Suzuki, M., Ishioka, N., Ohmori, H. and Honma. M. (2008b) Repair of I-Scel Induced DSB at a specific site of chromosome in human cells: influence of low-dose, low-dose rate gamma-rays, Radiat. Environ. Biophys., 47, 439-444.

Zhou, P.K., Liu, X.Y., Sun, W.Z., Zhang, Y.P. and Wei, Y.P.K. (1993) Cultured mouse SR-1 cells exposed to low-dose of $\gamma$-rays become less susceptible to the induction of mutations by radiation as well as bleomycin, Mutagenesis, 8, 109-111. 